\title{
Development and Cross-Validation of a Nomogram for Chronic Kidney Disease Following Robot-Assisted Radical Cystectomy
}

\author{
Ahmed S. Elsayed, MD, Zhe Jing, MA, Deniz Demirbas, PA, Mohammad Durrani, BS, \\ Kristopher Attwood, PhD, Joseph Cilento, BA, Jennifer A. Osei, BS, Sean Gibson, BS, Michael Mostowy, BS, \\ Amylisa Christophe, MSMT, Ahmed A. Hussein, MD, and Khurshid A. Guru, MD
}

\begin{abstract}
Introduction: We sought to identify the factors associated with deterioration of renal functions after robotassisted radical cystectomy, and to develop a nomogram to detect the probability of progression to chronic kidney disease (CKD).

Materials and Methods: A retrospective review of our prospectively maintained database. Estimated glomerular filtration rate (eGFR) was calculated using the CKD-Epidemiology Collaboration creatinine formula utilizing all follow-up creatinine values. CKD was defined as stage $3 \mathrm{~b}\left(\mathrm{eGFR}<45 \mathrm{~mL} / \mathrm{minute} / 1.73 \mathrm{~m}^{2}\right)$ based on the National Kidney Foundation classification. Kaplan-Meier curves were used to depict CKD-free survival. A multivariate Cox regression model was used to determine predictors for CKD and to build the perioperative nomogram.

Results: The data set comprised 442 patients with a median follow-up of 25 months (12-59). Thirty-seven percent developed CKD at a median of 9 months (4-18). CKD-free survival rates at 1,3, and 5 years were $75 \%, 58 \%$, and $50 \%$, respectively. CKD was significantly associated with preoperative eGFR (hazards ratio [HR]: 0.96, 95\% confidence interval [CI]: 0.95-0.97, $p<0.01$ ), body mass index (HR: 1.03, 95\% CI: $1.01-1.05, p=0.03$ ), Charlson Comorbidity Index $\geq 3$ (HR: 2.20, 95\% CI: 1.35-3.58, $p<0.01$ ), diabetes (HR: 1.59, 95\% CI: 1.09-2.31, $p=0.02$ ), 90 days postoperative strictures (HR: 4.04, 95\% CI: 1.76-9.30, $p<0.01$ ), 90 days postoperative hydronephrosis (HR: 2.26, 95\% CI: 1.34-3.79, $p<0.01$ ), 90 days recurrent urinary tract infection (HR: 1.84, 95\% CI: 1.08-3.14, $p=0.02$ ), 90 days acute kidney injury (HR: $1.70,95 \%$ CI: $1.19-2.43, p<0.01$ ), and node positive disease (HR: 1.94, 95\% CI: 1.31-2.86, $p<0.01$ ). A 5-year CKD-free survival nomogram was developed.

Conclusion: We have developed and cross-validated a nomogram for detecting CKD-free survival. This nomogram may have a role in counseling and follow up of patients. This study was done after the approval of the IRB committee (I-79606).
\end{abstract}

Keywords: radical cystectomy, robot assisted, chronic kidney disease, nomogram, renal function

\section{Introduction}

B LADDER CANCER IS the ninth-most common tumor worldwide with 81,190 estimated new cases and 17,240 estimated deaths in the United States in 2018. ${ }^{1}$ Radical cystectomy (RC) with pelvic lymphadenectomy and urinary diversion is the standard of care for surgical management of organ-confined muscle invasive bladder cancer and refractory nonmuscle invasive disease. ${ }^{2}$ Robot-assisted radical cystectomy (RARC) has risen from $0.6 \%$ in 2004 to $32.4 \%$ of cystectomies in 2017 in the United States. ${ }^{3}$ RARC has pro- vided equivalent early oncologic outcomes to open $\mathrm{RC}$, and probably superior when it comes to perioperative outcomes. ${ }^{4}$ However, RC remains a morbid procedure with high complication rate, most of which are diversion related..$^{5}$ Among the long-term complications of urinary diversion is deterioration of renal function. ${ }^{6,7}$

Chronic kidney disease (CKD) is associated with higher risk of cardiovascular disease, immunodeficiency, prolonged hospitalization, as well as recurrence and mortality rate. ${ }^{8,9}$ While bladder cancer is one of the most expensive cancers to treat, the presence of CKD may pose an additional health care 
and economic burden. ${ }^{10}$ Nomograms have been widely accepted by physicians as tools for assessing patient prognosis. We sought to identify the factors associated with deterioration of renal functions after RARC, and to develop a nomogram to detect the probability of progression to $\mathrm{CKD}$.

\section{Materials and Methods}

A retrospective review of our quality assurance institutional database of 630 patients who underwent RARC between 2005 and 2019 was performed (I-79606). Patients with incomplete data, preoperative CKD (estimated glomerular filtration rate $[\mathrm{eGFR}]<45 \mathrm{~mL} / \mathrm{minute} / 1.73 \mathrm{~m}^{2}$ ), or $<3$ months of follow-up were excluded (Fig. 1). Data were reviewed for preoperative characteristics, operative, and postoperative outcomes. Patients were divided into those who developed CKD [defined as stage $3 \mathrm{~b}\left(<45 \mathrm{~mL} /\right.$ minute $\left./ 1.73 \mathrm{~m}^{2}\right)$ based on the National Kidney Foundation classification at least 90 days after RARC] and those who did not.

eGFR was computed using the Chronic Kidney Disease Epidemiology Collaboration (CKD-EPI) creatinine formula utilizing all follow-up creatinine values (excluding the first 3 months post-RARC). Acute kidney injury (AKI) was defined as an abrupt (within 48 hours) reduction in kidney function, defined as an absolute increase in serum creatinine of more than or equal to $0.3 \mathrm{mg} / \mathrm{dL}(\geq 26.4 \mu \mathrm{mol} / \mathrm{L})$, or a percentage increase in serum creatinine of more than or equal to $50 \%$ (1.5-fold from baseline). ${ }^{11}$ Urinary tract infections (UTIs) were defined as the presence of positive urine culture ( $\geq 10^{5} \mathrm{cfu} / \mathrm{mL}$ with no more than two organisms) and documented urinary symptoms, including fever $\left(\geq 38^{\circ} \mathrm{C}\right)$, chills, flank, or abdominal pain.

Data were analyzed with double-sided t-test and Wilcoxon test and described in terms of means \pm standard deviations

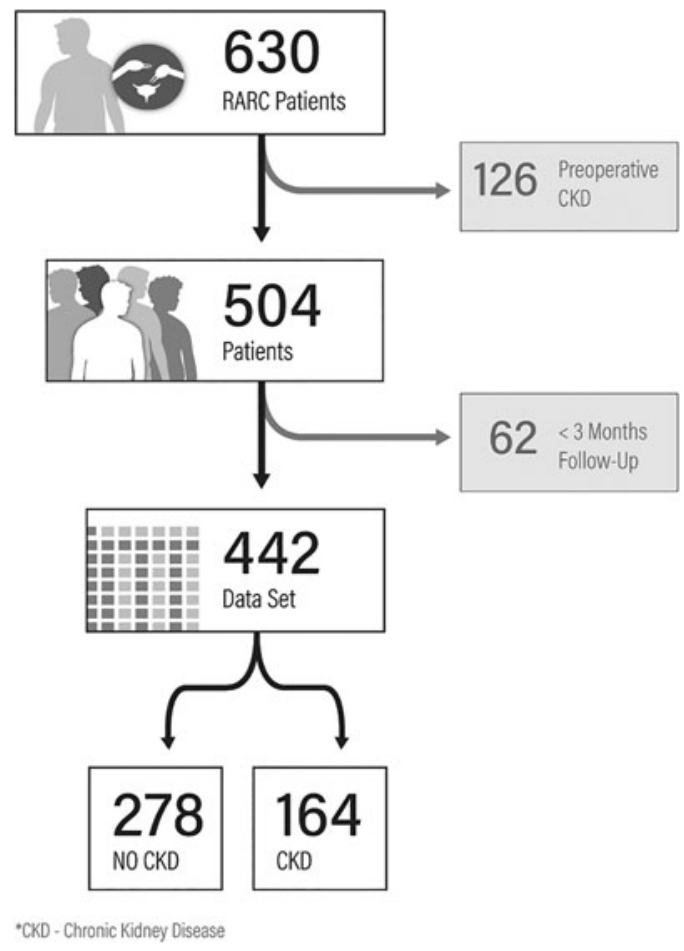

FIG. 1. Flowchart of patient selection.
(SDs) and median (interquartile range [IQR]). Multivariable stepwise Cox regression model was used to depict predictors of CKD. The Kaplan-Meier method was used to compute CKD. After 90 days, an eGFR $<45 \mathrm{~mL} /$ minute $/ 1.73 \mathrm{~m}^{2}$ was considered an event; the duration from day 90 to the date of event/censor is defined as survival time. The nomogram prognosticators included preoperative, operative, and 90-day postoperative variables based on the multivariate model. The nomogram was constructed to provide visual explanation for predicted probabilities of a survival outcome, as obtained by the statistical predictive model. The point system was carried out in scoring based on the weighted relative importance of individual risk factors, and individual 1-, 2-, 3-, 4-, and 5-year CKD-free survival probability.

The utility of the nomogram was evaluated using timedependent receiver operating characteristic (ROC) curves, area under the curve (AUC), calibration plots, and prediction errors. Standard cross-validation techniques were used to generate these measures. All tests were two sided, with statistical significance defined as $p \leq 0.05$. All statistical analyses were performed using SAS ${ }^{\circledR}$ software (version 9.4; SAS Institute, Inc., Cary, $\mathrm{NC}$ ) and $\mathrm{R}^{\circledR}$ software 3.6.1.

\section{Results}

The final cohort included 442 patients with a median follow-up of 25 months (IQR 12-59). Thirty-seven percent developed CKD at a median time of 9 months (IQR 4-18). Patients who developed CKD were older $(70 \pm 9$ years vs $66 \pm 11$ years, $p<0.01$ ), had Charlson Comorbidity Index $(\mathrm{CCI}) \geq 3$ more frequently $(87 \%$ vs $73 \%, p<0.01)$, and had a lower mean preoperative eGFR $\left(68 \mathrm{~mL} / \mathrm{minute} / 1.73 \mathrm{~m}^{2} \mathrm{vs}\right.$ $\left.82 \mathrm{~mL} / \mathrm{minute} / 1.73 \mathrm{~m}^{2}, p<0.01\right)$. There was no statistical difference between the groups with regard to body mass index (BMI) $(p=0.08)$, race $(p=0.48)$, American Society of Anesthesiology (ASA) $\geq 3(p=0.69)$, receipt of neoadjuvant chemotherapy (NAC) $(p=0.12)$, rate of intracorporeal urinary diversion $(p=0.73)$, ileal conduit urinary diversion $(p=0.54)$, estimated blood loss $(p=0.26)$, and operative time $(p=0.86)$.

Patients who developed CKD experienced a higher rate of overall $(91 \%$ vs $80 \%, p<0.01)$ and high-grade complications (54\% vs $27 \%, p<0.01)$. There was no significant difference in terms of $\geq \mathrm{pT} 3(36 \%$ vs $31 \%, p=0.40)$, positive soft tissue surgical margins ( $4 \%$ vs $6 \%, p=0.28)$, node-positive disease $(\mathrm{pN}+\mathrm{ve})(22 \%$ vs $19 \%, p=0.54)$, and recurrence rates $(29 \%$ vs 26\%, $p=0.51$ ) (Table 1).

CKD-free survival rates at 1, 3, and 5 years were $75 \%$, $58 \%$, and $50 \%$, respectively (Fig. 2). CKD was significantly associated with preoperative eGFR (hazards ratio [HR]: 0.96, 95\% confidence interval $[\mathrm{CI}]: 0.95-0.97, p<0.01)$, BMI (HR: 1.03, 95\% CI: 1.01-1.05, $p=0.03$ ), CCI $\geq 3$ (HR: 2.20 , 95\% CI: $1.35-3.58, p<0.01$ ), diabetes (HR: 1.59 , 95\% CI: 1.09-2.31, $p=0.02)$, 90-day postoperative ureteroileal strictures (HR: 4.04, 95\% CI: 1.76-9.30, $p<0.01$ ), 90-day postoperative hydronephrosis (HR: 2.26, 95\% CI: 1.34-3.79, $p<0.01$ ), 90-day recurrent UTI (HR: 1.84, 95\% CI: $1.08-$ $3.14, p=0.02)$, 90-day AKI (HR: 1.70, $95 \%$ CI: 1.19-2.43, $p<0.01$ ), and $\mathrm{pN}+\mathrm{ve}(\mathrm{HR}: 1.94,95 \% \mathrm{CI}: 1.31-2.86, p<0.01)$ (Table 2). A 5-year CKD-free survival nomogram was developed based on these predictors (Fig. 3). The strongest predictor for CKD was baseline eGFR. 
Table 1. Baseline and Perioperative Characteristics

\begin{tabular}{|c|c|c|c|c|}
\hline Perioperative parameters & No $C K D$ & $C K D$ & All & $\mathrm{p}$ \\
\hline No. of patients (\%) & $278(63)$ & $164(37)$ & $442(100)$ & \\
\hline Age at cystectomy, mean \pm SD (year) & $66 \pm 11$ & $70 \pm 9$ & $68 \pm 10$ & $<0.01$ \\
\hline Gender, male $n(\%)$ & $212(76)$ & $116(71)$ & $328(74)$ & 0.22 \\
\hline White race, $n(\%)$ & $266(96)$ & $154(94)$ & $420(95)$ & 0.48 \\
\hline BMI, mean $\pm \mathrm{SD}\left(\mathrm{kg} / \mathrm{m}^{2}\right)$ & $29 \pm 7$ & $30 \pm 6$ & $29 \pm 6$ & 0.08 \\
\hline ASA score $\geq 3, n(\%)$ & $141(54)$ & $81(52)$ & $222(53)$ & 0.69 \\
\hline $\mathrm{CCI} \geq 3, n(\%)$ & $204(73)$ & $143(87)$ & 347 (79) & $<0.01$ \\
\hline Prior abdominal/pelvic surgery, $n(\%)$ & $153(55)$ & $94(57)$ & $247(56)$ & 0.69 \\
\hline Prior irradiation, $n(\%)$ & $16(6)$ & $10(6)$ & $26(6)$ & 1 \\
\hline NAC, $n(\%)$ & $69(25)$ & $52(32)$ & $121(27)$ & 0.12 \\
\hline Type of diversion, ileal conduit, $n(\%)$ & $240(86)$ & $144(88)$ & $384(87)$ & 0.54 \\
\hline ICUD, $n(\%)$ & $204(75)$ & $124(77)$ & $328(75)$ & 0.73 \\
\hline Operative time, median (IQR) (minutes) & $360(300-431)$ & $366(298-434)$ & $362(300-433)$ & 0.86 \\
\hline Estimated blood loss, median (IQR) (mL) & $200(150-400)$ & $250(150-475)$ & $250(150-400)$ & 0.26 \\
\hline Blood transfusion, $n(\%)$ & $12(4)$ & $10(6)$ & $22(5)$ & 0.5 \\
\hline Any complication, $n(\%)$ & $222(80)$ & $150(91)$ & $372(84)$ & $<0.01$ \\
\hline High-grade complications, $n(\%)$ & $76(27)$ & $89(54)$ & $165(37)$ & $<0.01$ \\
\hline Hospital stay, median (IQR) (days) & $7(6-9)$ & $7(6-11)$ & $7(6-9)$ & 0.33 \\
\hline 90-day adjuvant chemotherapy, $n(\%)$ & $18(6)$ & $15(9)$ & $33(7)$ & 0.35 \\
\hline Adjuvant chemotherapy, $n(\%)$ & $41(15)$ & 31 (19) & $72(16)$ & 0.29 \\
\hline$\geq \mathrm{pT} 3, n(\%)$ & $87(31)$ & $58(36)$ & $145(33)$ & 0.40 \\
\hline +ve soft tissue surgical margins, $n(\%)$ & $18(6)$ & $6(4)$ & $24(5)$ & 0.28 \\
\hline $\mathrm{pN}+\mathrm{ve}, n(\%)$ & $53(19)$ & $35(22)$ & $88(20)$ & 0.54 \\
\hline Any recurrence, $n(\%)$ & $71(26)$ & $47(29)$ & $118(27)$ & 0.51 \\
\hline
\end{tabular}

Boldface indicates significant values.

$\mathrm{BMI}=$ body mass index; $\mathrm{CCI}=$ Charlson Comorbidity Index; $\mathrm{CKD}=$ chronic kidney disease; $\mathrm{IQR}=$ interquartile range; $\mathrm{NAC}=$ neoadjuvant chemotherapy; $\mathrm{pN}+\mathrm{ve}=$ node positive disease; $\mathrm{SD}=$ standard deviation .

FIG. 2. Kaplan-Meier curve showing CKD-free survival. $\mathrm{CKD}=$ chronic kidney disease.
Product-Limit Survival Estimate

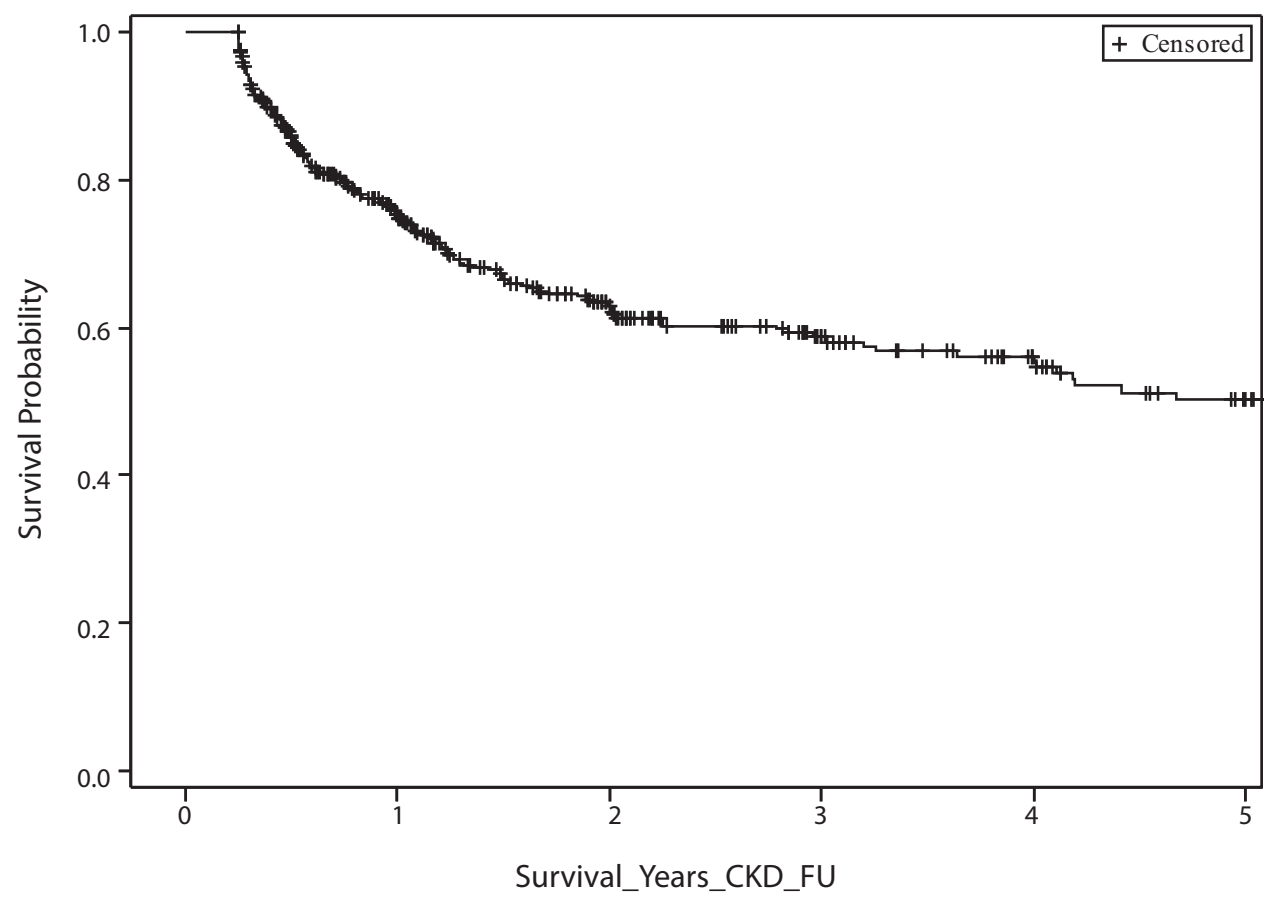

\begin{tabular}{|l|c|c|c|c|c|}
\hline Years & $\mathbf{1}$ & $\mathbf{2}$ & $\mathbf{3}$ & $\mathbf{4}$ & $\mathbf{5}$ \\
\hline Survival Rate \% & 75 & 63 & 58 & 56 & 50 \\
\hline Failed & 100 & 36 & 9 & 4 & 7 \\
\hline Sample Size & 442 & 251 & 150 & 99 & 74 \\
\hline
\end{tabular}


Table 2. Multivariate Analysis Predicting Chronic Kidney Disease

\begin{tabular}{lccr}
\hline Variable & $\begin{array}{c}\text { Hazards } \\
\text { ratio }\end{array}$ & $\begin{array}{c}\text { 95\% confidence } \\
\text { interval }\end{array}$ & $\mathrm{p}$ \\
\hline Preoperative eGFR & 0.96 & $0.95-0.97$ & $<0.01$ \\
BMI & 1.03 & $1.01-1.05$ & 0.03 \\
CCI $\geq 3$ & 2.20 & $1.35-3.58$ & $<0.01$ \\
Diabetes mellitus & 1.59 & $1.09-2.31$ & 0.02 \\
90-day ureteroileal & 4.04 & $1.76-9.30$ & $<0.01$ \\
$\quad$ strictures & & & \\
90-day hydronephrosis & 2.26 & $1.34-3.79$ & $<0.01$ \\
90-day recurrent UTI & 1.84 & $1.08-3.14$ & 0.02 \\
90-day AKI & 1.70 & $1.19-2.43$ & $<0.01$ \\
pN+ve & 1.94 & $1.31-2.86$ & $<0.01$ \\
\hline
\end{tabular}

$\mathrm{AKI}=$ acute kidney injury; $\mathrm{eGFR}=$ estimated glomerular filtration rate; $\mathrm{UTI}=$ urinary tract infection.

The quantified ROC curve was plotted, and the AUC at year 1 to 5 was $0.77,0.79,0.77,0.77$, and 0.78 , respectively (Fig. 4). Calibration plots for CKD-free survival probability at all 5 years showed acceptable calibration, and the predictions reasonably approximated actual survival probabilities.
On average, our model slightly underestimates the CKD-free survival rates by less than $0.5 \%$; the $\mathrm{SD}$ of prediction error is controlled to a range of $2.6 \%$ to $4.2 \%$ (Fig. 5).

The use of the nomogram is best illustrated with an example. Assume a patient has been diagnosed with refractory nonmuscle invasive bladder cancer and he underwent RARC after patient counseling. The patient has a baseline eGFR of 90, and BMI of 35 . He did not receive NAC. He developed postoperative stricture, hydronephrosis, and recurrent UTI. At the 3-month follow-up, the patient will be counseled regarding his CKD-free survival. His scores based on the nomogram will be $44,15,43,25$, and 19 , respectively. The sum of the scores is 146 points. The CKD-free survival for this patient based on the nomogram is $50 \%$ at 1 year and $10 \%$ at 5 years (Fig. 6).

\section{Discussion}

Approximately $70 \%$ of patients undergoing RC with urinary diversion experience renal function decline regardless of type of diversion. ${ }^{12}$ The presence of CKD significantly affects the management of bladder cancer patients, alters decisions for treatment, and can also affect patients' prognosis. CT imaging with contrast is the mainstay for diagnosing and follow up of bladder cancer. CKD may hinder patients from

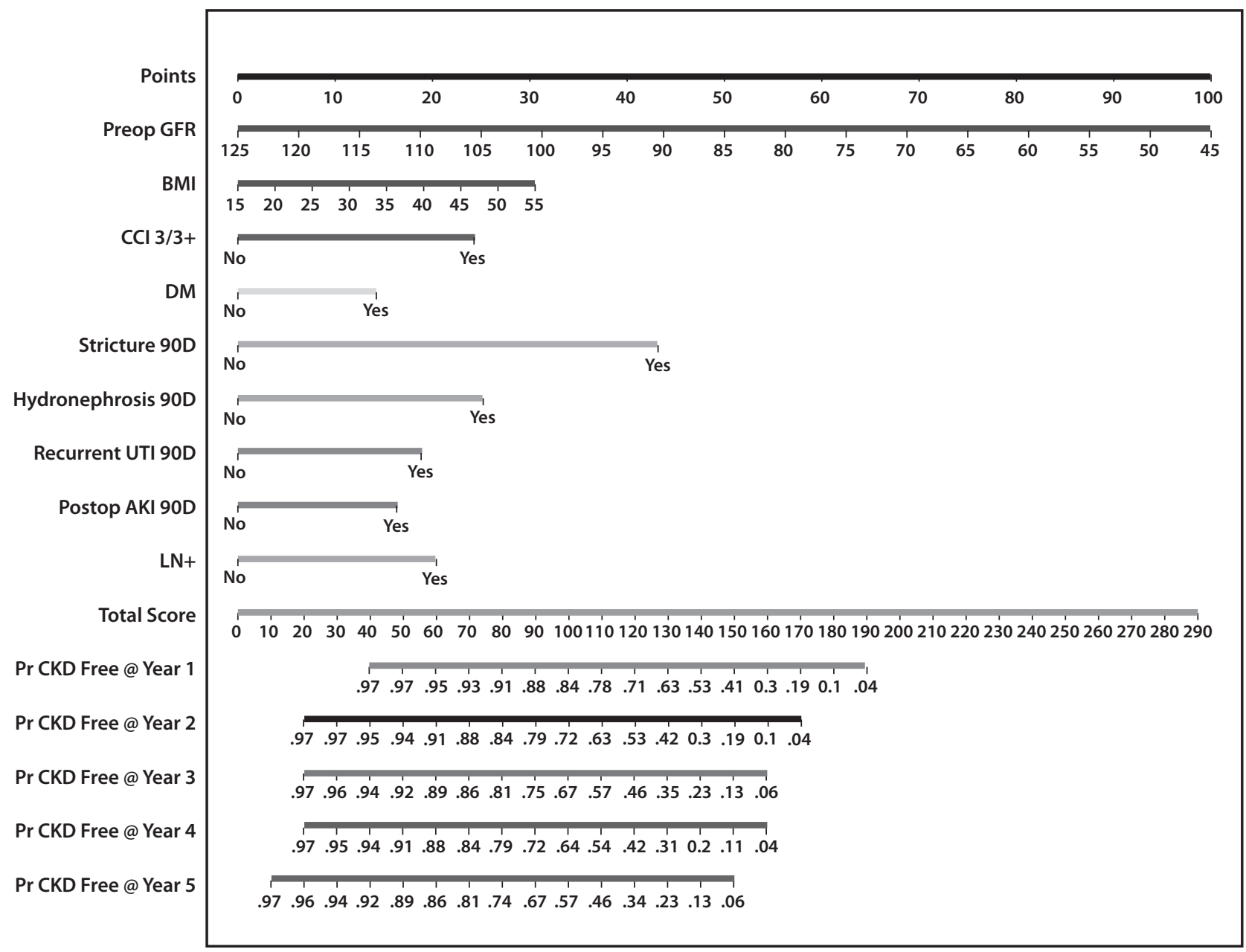

FIG. 3. Nomogram for prediction of chronic kidney disease after RARC. PR=predicted; RARC=robot-assisted radical cystectomy. 

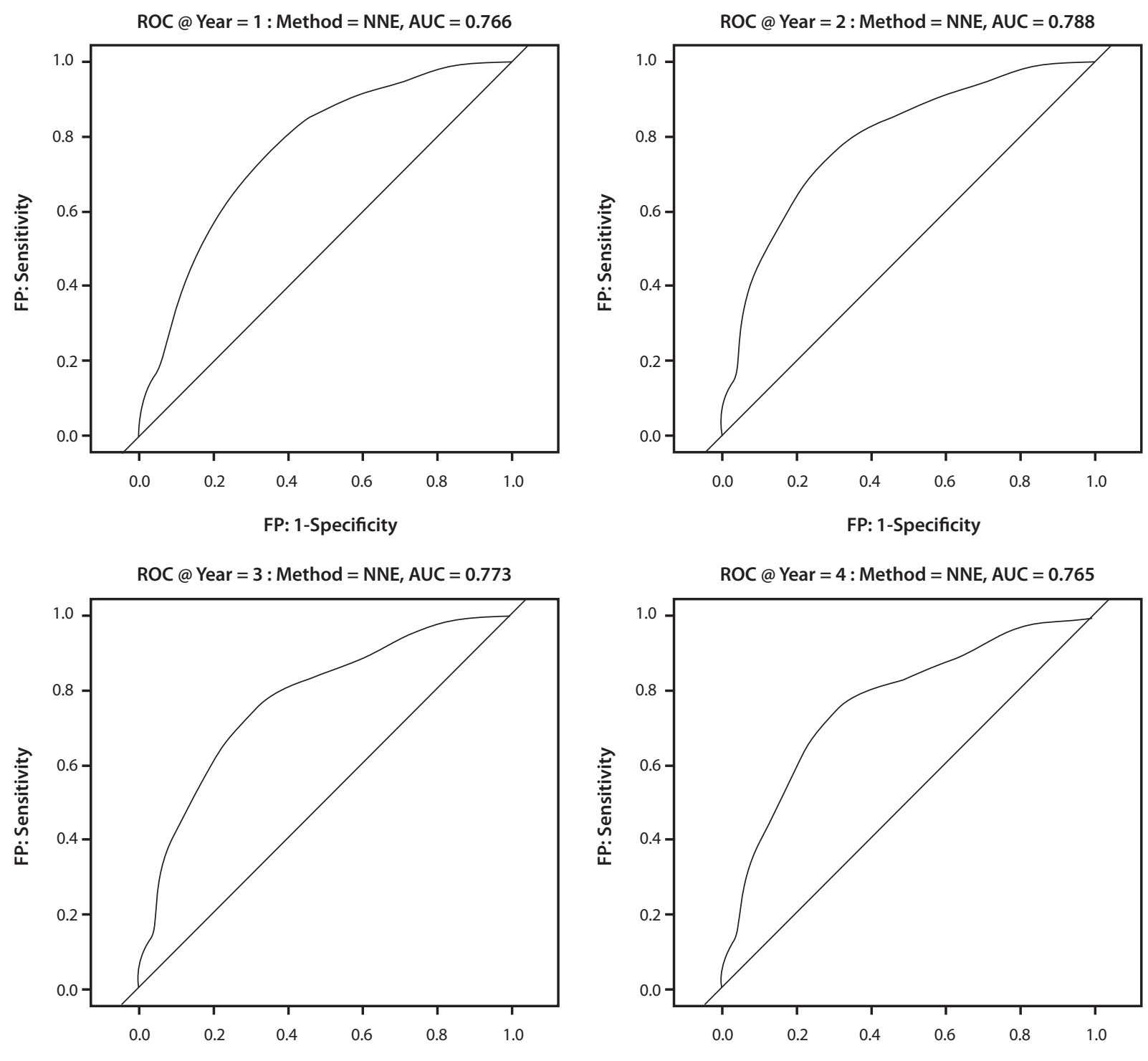

FP: 1-Specificity

FP: 1-Specificity

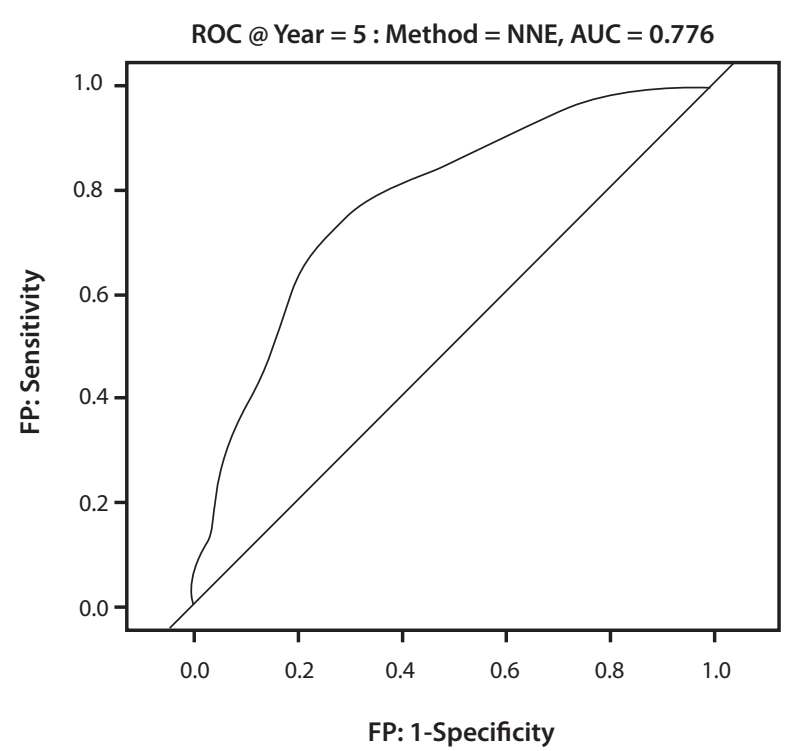

FIG. 4. Receiver operation curve showing the accuracy of the nomogram at years $1,2,3,4$, and 5 , respectively. $\mathrm{FP}=$ false positive. 


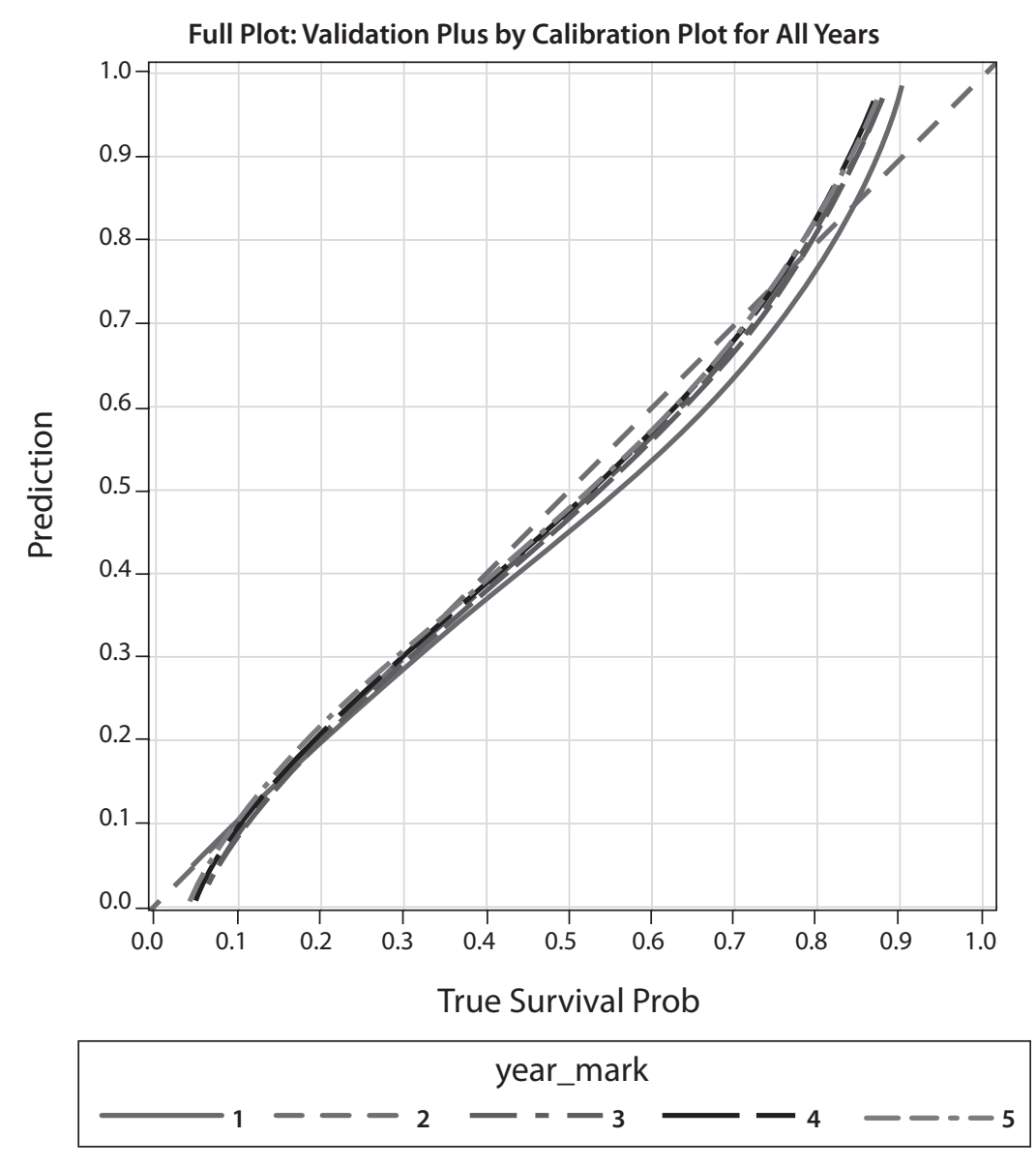

FIG. 5. Calibration plots for estimating CKD-free survival at 1, 2, 3, 4, and 5 years.

\begin{abstract}
The red dotted line is the reference line that indicates where a perfect calibration would lie. The prediction errors of year 1 to year 5 were $-0.5 \% \pm 4.2 \%,-0.3 \% \pm 3.6 \%$,
\end{abstract} $-0.3 \% \pm 3.2 \%,-0.29 \% \pm 3.0 \%,-0.3 \% \pm 2.6 \%$, respectively.

receiving CT scans. CKD will deem the patient ineligible for chemotherapy whether neoadjuvant or adjuvant. ${ }^{13}$ Significant reduction in kidney function is considered by many an absolute contraindication for continent urinary diversion; although some studies (including the current) did not find a significant association between the type of diversion and probability of CKD. ${ }^{2,14}$ A systematic review and metaanalysis found that preoperative CKD is associated with a significantly worse recurrence-free, disease-specific, and overall survival. ${ }^{15}$ Another has shown that CKD was an independent risk factor for bladder/upper urinary tract tumor recurrences and overall survival among patients with nonmuscle invasive bladder cancer. Thus, preservation of renal function should be one of the primary goals after RC and urinary diversion. Previous reports have studied several predictors for poor renal function after RC. ${ }^{16}$ However, there is a lack of nomograms to predict the probability of CKD after RARC. In this study, we developed a nomogram to predict long-term renal functions for patients undergoing RARC.

Previous studies have used serum creatinine, nuclear imaging, and eGFR to calculate renal function. We have used the eGFR based on the CKD-EPI creatinine formula, which was proven accurate and comparable with the Modification of Diet in Renal Disease formula especially in older age $(>65) .{ }^{17}$ There is lack of a standardized definition for renal function deterioration after RC. A study that has looked into long-term renal function after $\mathrm{RC}$ has defined decreased renal function as a decrease in eGFR $>10 \mathrm{~mL} /$ minute $/ 1.73 \mathrm{~m}^{2} .{ }^{16}$ Hautmann and colleagues defined a $25 \%$ decline in eGFR from baseline as renal function decline, and Jin and colleagues and Eisenberg and colleagues defined the decline as a $10 \%$ decline in eGFR from baseline. ${ }^{16,18,19}$ It is evident that a certain level of decline in renal function is expected after urinary diversion, but no clear evident threshold value has been determined for renal function decline after urinary diversion. We chose CKD stage $3 \mathrm{~b}$ as a threshold as this was associated with worse progression-free, cancer-specific, and overall survival. ${ }^{20}$

Prevalence of CKD after RC varies widely in the literature. In the Mayo Clinic series, $20 \%$ of patients undergoing RC progressed to CKD (stage III-V) after a median follow-up of 5.8 years. $^{21}$ Mayo Clinic did not exclude patients with preoperative $\mathrm{CKD}$, which could explain the lower percentage of progression as these patients probably received an extensive nephrologic evaluation and follow-up. Jin and colleagues have demonstrated an incidence rate of CKD (stage III-V) of 


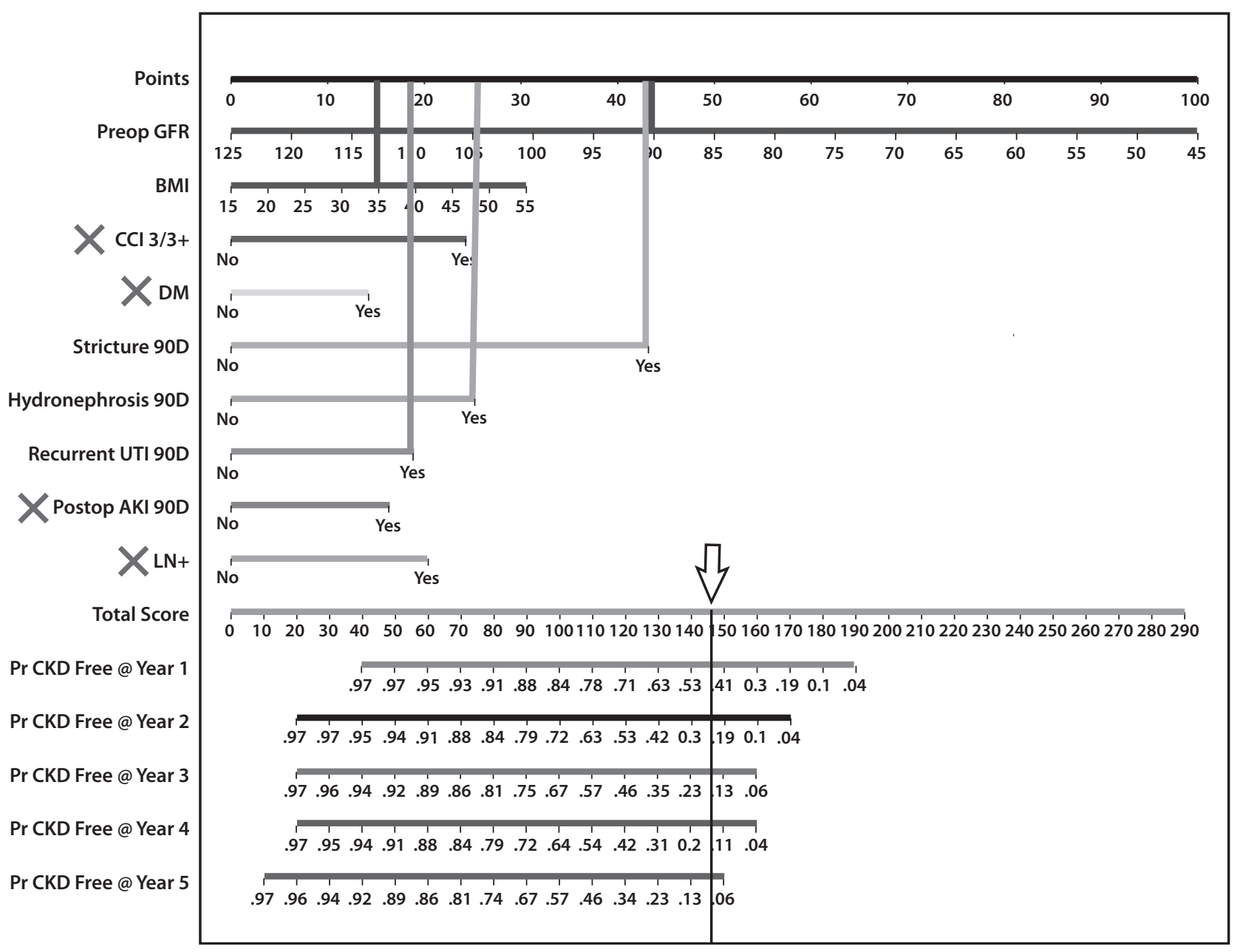

FIG. 6. Example of how to use the nomogram.

$26 \%$ and $17 \%$ for ileal conduit and orthotopic urinary diversions, respectively, at 10 or more years after surgery. ${ }^{16}$ Our CKD incidence rate is $37 \%$, which was defined as stage $\geq 3 \mathrm{~b}$ compared with Jin and colleagues, which was defined as stage $\geq 3$ a.

The importance of baseline eGFR is well established for patients undergoing partial nephrectomies. This might be attributed to the presence of the inevitable impact of aging and medical comorbidities, which is supported by the findings of Simmons and colleagues showing that the kidney tissue quality, as measured by baseline eGFR, has a significant effect on the long-term renal function outcomes after partial nephrectomy. ${ }^{22}$ Baseline eGFR was the strongest predictor for CKD-free survival in our nomogram as suggested by previous extensive literature for partial nephrectomies.

BMI, CCI $\geq 3$, and diabetes were all significant in our model. Obesity is often associated with diabetes and hypertension as a part of the metabolic syndrome. Studies have shown that obesity is a preventable risk factor for renal function deterioration and although hypertension and type 2 diabetes are important mediators, additional pathways may exist. ${ }^{23}$ Higher BMI increases the complexity of the procedure, which may increase the operative time, predisposing to AKI, which also increases the risk of CKD. ${ }^{24}$ Diabetes mellitus is a wellestablished risk factor for CKD. ${ }^{25}$ Our study agrees with the literature and further highlights the importance of optimization of comorbid conditions, especially diabetes control for renoprotection. AKI is associated with a significant risk of CKD (HR: 1.91). ${ }^{26}$ Also, postoperative AKI is associated with increased morbidity, mortality rate, and length-of-hospital stay following many types of major surgeries. ${ }^{27}$ The incidence of AKI after open RC is $11 \%-33 \% .^{28}$ Predictors of AKI were $\mathrm{BMI}$, preoperative plasma creatinine, intraoperative blood loss, volume of administered crystalloids, fluid balance, and duration of surgery. ${ }^{28}$ Optimization of the patient condition and adhering to the enhanced recovery after surgery principles may help reduce the incidence of AKI.

A previous work from our group reported a ureteroileal stricture rate after RARC of $13 \%$. More than two-thirds of the strictures developed in asymptomatic patients, which may delay the diagnosis, management, and subsequently exacerbate renal injury. ${ }^{29} \mathrm{Jin}$ and colleagues have looked at the longterm deterioration of renal function after RC and they concluded that obstruction is the single most important cause of renal function deterioration despite the diversion type. ${ }^{16} \mathrm{~A}$ propensity score-matched study looking at renal function deterioration between different types of diversion identified hydronephrosis, strictures, and stones as significant predictors. ${ }^{14}$ Strictures, hydronephrosis, and recurrent UTI may correlate significantly, however, our nomogram has highlighted the 
importance of each of them. Implanting the ureters in a refluxing manner causes minimal pelvicaliceal system dilatation, which could be confused sometimes with strictures causing mild obstruction. The latter may be asymptomatic in $75 \%$, but can cause gradual loss of renal function over time. ${ }^{29}$ The presence of higher grade hydronephrosis indicates more severe obstruction due to stricture disease, and a superimposed infection can dramatically worsen the kidney functions. UTI occurs in up to $36 \%$ of patients after RC, especially with the presence of hydronephrosis and/or ureteroileal strictures, and chronic UTIs have been associated with deterioration of renal functions after RC. ${ }^{16,30}$ This highlights the importance of regular follow-up for early detection and management of postoperative complications.

$\mathrm{pN}+\mathrm{ve}$ may be attributed to stricture formation and hydronephrosis. $\mathrm{pN}+\mathrm{ve}$ disease will require more aggressive lymph node and ureteral dissection, which may ultimately lead to more ureteroileal strictures. Patients with $\mathrm{pN}+\mathrm{ve}$ disease may receive adjuvant chemotherapy, which is nephrotoxic. The contribution of adjuvant chemotherapy in this study may be underestimated, as we focused more on the preoperative, operative, and immediate 90 -day postoperative variables associated with CKD. Adjuvant chemotherapy in many cases is delayed until patients fully recover from surgery and can tolerate chemotherapy.

NAC was not a predictor of CKD in our nomogram despite the known effect of chemotherapy on renal functions. This may be explained by patients with refractory nonmuscle invasive bladder cancer (45\%) undergoing RARC. Also, the low utilization rate of NAC (27\%) in our cohort. Both factors might affect the importance of NAC in our model.

Whether the type of urinary diversion affects renal functions after RC or not remains a matter of debate. Our model, as well as prior studies, has shown no difference between ileal conduits and neobladders. ${ }^{14}$ However, other retrospective studies have shown opposite results. ${ }^{16}$ Patients who receive ileal conduits tend to be older with multiple comorbidities at baseline, which may further worsen their kidney function. On the contrary, continent urinary reservoirs allow for more contact time between the urine and the intestine, which leads to increased absorption of electrolytes that can ultimately compromise renal function. ${ }^{7}$

The importance of our nomogram is that it will aid in patient counseling about the future risk of developing CKD. Our nomogram also highlights the importance of preparing the patients medically and psychologically so that they are not blind to the outcome of their disease. Furthermore, getting a nephrologist early onboard adds diversity of thought to care. The choice of urinary diversion approach and follow-up protocols may be altered based on the patient's specific probability of CKD.

To our knowledge, this is the largest series investigating CKD-free survival after RARC and developing a nomogram. However, some limitations do exist. Although data were collected prospectively, our results are still subjected to the limitations and biases associated with retrospective analysis. This is a single-center study and external validation is required. All patients included had undergone RARC, and no ORC cases were included. Despite this, we do not believe that an open approach will pose a significant difference in prediction of renal function. The aforementioned variables rather than the technique are related to renal functions. Proteinuria was not captured for any of the patients, which may have a prognostic effect. Also, the hydration status at the time of creatinine withdrawal was not reported. A physiologic decrease in eGFR per year was not corrected for. The improvement of eGFR after the relief of obstruction was not captured. ${ }^{16}$ External validation of the model is warranted before use in clinical practice.

\section{Conclusion}

We have developed and cross-validated a nomogram to estimate the CKD-free survival up to 5 years post-RARC, based on nine objective and easy to acquire variables.

\section{Author Disclosure Statement}

No competing financial interests exist.

\section{Funding Information}

No funding was received for this article.

\section{References}

1. Siegel RL, Miller KD, Jemal A. Cancer statistics, 2018. CA Cancer J Clin 2018;68:7-30.

2. Alfred Witjes J, Lebret T, Compérat EM, et al. Updated 2016 EAU Guidelines on Muscle-invasive and metastatic bladder cancer. Eur Urol 2017;71:462-475.

3. Gill I, Cacciamani G. The changing face of urologic oncologic surgery from 2000-2018 (63 141 patients)-impact of robotics. Eur Urol Suppl 2019;18:e656-e7.

4. Tyritzis SI, Collins JW, Wiklund NP. The current status of robot-assisted cystectomy. Indian J Urol 2018;34:101.

5. Johar RS, Hayn MH, Stegemann AP, et al. Complications after robot-assisted radical cystectomy: Results from the International Robotic Cystectomy Consortium. Eur Urol 2013;64:52-57.

6. Hautmann RE, de Petriconi RC, Volkmer BG. 25 years of experience with 1,000 neobladders: Long-term complications. J Urol 2011;185:2207-2212.

7. Lawrentschuk N, Colombo R, Hakenberg OW, et al. Prevention and management of complications following radical cystectomy for bladder cancer. Eur Urol 2010;57:983-1001.

8. Go AS, Chertow GM, Fan D, McCulloch CE, Hsu C-y. Chronic kidney disease and the risks of death, cardiovascular events, and hospitalization. N Engl J Med 2004;351:1296-1305.

9. Thomas R, Kanso A, Sedor JR. Chronic kidney disease and its complications. Prim Care 2008;35:329-344.

10. Sloan FA, Yashkin AP, Akushevich I, Inman BA. The cost to medicare of bladder cancer care. Eur Urol Oncol 2019. [Epub ahead of print]; DOI: 10.1016/j.euo.2019.01.015.

11. Mehta RL, Kellum JA, Shah SV, et al. Acute Kidney Injury Network: Report of an initiative to improve outcomes in acute kidney injury. Crit Care 2007;11:R31.

12. Gershman B, Eisenberg MS, Thompson RH, et al. Comparative impact of continent and incontinent urinary diversion on long-term renal function after radical cystectomy in patients with preoperative chronic kidney disease 2 and chronic kidney disease 3a. Int J Urol 2015;22:651-656.

13. Milowsky MI, Rumble RB, Booth CM, et al. Guideline on muscle-invasive and metastatic bladder cancer (European Association of Urology guideline): American Society of Clinical Oncology clinical practice guideline endorsement. J Clin Oncol 2016;34:1945-1952.

14. Hatakeyama S, Koie $T$, Narita $T$, et al. Renal function outcomes and risk factors for risk factors for stage $3 \mathrm{~B}$ chronic kidney disease after urinary diversion in patients 
with muscle invasive bladder cancer. PLoS One 2016;11: e0149544.

15. Cao J, Zhao X, Zhong Z, Zhang L, Zhu X, Xu R. Prognostic value of pre-operative renal insufficiency in urothelial carcinoma: A systematic review and meta-analysis. Sci Rep 2016;6:1-9.

16. Jin X-D, Roethlisberger S, Burkhard FC, Birkhaeuser F, Thoeny HC, Studer UE. Long-term renal function after urinary diversion by ileal conduit or orthotopic ileal bladder substitution. Eur Urol 2012;61:491-497.

17. Flamant M, Haymann J-P, Vidal-Petiot E, et al. GFR estimation using the Cockcroft-Gault, MDRD study, and CKD-EPI equations in the elderly. Am J Kidney Dis 2012; 60:847-849.

18. Hautmann RE, Abol-Enein H, Hafez K, et al. Urinary diversion. Urology 2007;69:17-49.

19. Eisenberg MS, Thompson RH, Frank I, et al. Long-term renal function outcomes after radical cystectomy. J Urol 2014;191:619-625.

20. Matsumoto A, Nakagawa T, Kanatani A, et al. Preoperative chronic kidney disease is predictive of oncological outcome of radical cystectomy for bladder cancer. World J Urol 2018;36:249-256.

21. Kim S, Frank I, Weight C, et al. 1135 determinants of longterm renal function for patients undergoing radical cystectomy with urinary diversion. J Urol 2011;185(4S):e456-e.

22. Simmons MN, Fergany AF, Campbell SC. Effect of parenchymal volume preservation on kidney function after partial nephrectomy. J Urol 2011;186:405-410.

23. Ejerblad E, Fored CM, Lindblad P, Fryzek J, McLaughlin JK, Nyrén O. Obesity and risk for chronic renal failure. J Am Soc Nephrol 2006;17:1695-1702.

24. Hussein AA, May PR, Ahmed YE, et al. Development of a patient and institutional-based model for estimation of operative times for robot-assisted radical cystectomy: Results from the International Robotic Cystectomy Consortium. BJU Int 2017;120:695-701.

25. Association AD. Standards of medical care in diabetes2014. Diabetes Care 2014;37(Supplement 1):S14-S80.

26. Bucaloiu ID, Kirchner HL, Norfolk ER, Hartle II JE, Perkins RM. Increased risk of death and de novo chronic kidney disease following reversible acute kidney injury. Kidney Int 2012;81:477-485.

27. Goren O, Matot I. Perioperative acute kidney injury. Br J Anaesth 2015;115(suppl_2):ii3-ii14.

28. Furrer MA, Schneider MP, Burkhard FC, Wuethrich PY. Incidence and perioperative risk factors for early acute kidney injury after radical cystectomy and urinary diversion. Urol Oncol 2018;36:306.e17-306.e23.

29. Ahmed YE, Hussein AA, May PR, et al. Natural history, predictors and management of ureteroenteric strictures after robot assisted radical cystectomy. J Urol 2017;198:567574.

30. Ghoreifi A, Van Horn CM, Xu W, et al. Urinary tract infections following radical cystectomy with enhanced recovery protocol: A prospective study. Urol Oncol 2020;38: 75.e9-75.e14.

Address correspondence to: Khurshid A. Guru, MD

A.T.L.A.S (Applied Technology Laboratory for Advanced Surgery) Program Department of Urologic Oncology Roswell Park Comprehensive Cancer Center Elm and Carlton St Buffalo Buffalo, NY 14263 USA E-mail: khurshid.guru@ roswellpark.org

$\begin{aligned} & \text { Abbreviations Used } \\ \mathrm{AKI} & =\text { acute kidney injury } \\ \mathrm{ASA} & =\text { American Society of Anesthesiology } \\ \mathrm{AUC} & =\text { area under the curve } \\ \mathrm{BMI} & =\text { body mass index } \\ \mathrm{CCI} & =\text { Charlson Comorbidity Index } \\ \mathrm{CI} & =\text { confidence interval } \\ \mathrm{CKD} & =\text { chronic kidney disease } \\ \mathrm{CKD}-\mathrm{EPI} & =\text { chronic kidney disease epidemiology } \\ \mathrm{CT} & =\text { computed tomography } \\ \mathrm{eGFR} & =\text { estimated glomerular filtration rate } \\ \mathrm{HR} & =\text { hazards ratio } \\ \mathrm{IQR} & =\text { interquartile range } \\ \mathrm{RARC} & =\text { robot-assisted radical cystectomy } \\ \mathrm{RC} & =\text { radical cystectomy } \\ \mathrm{ROC} & =\text { receiver operating characteristic } \\ \mathrm{SD} & =\text { standard deviation } \\ \mathrm{NAC} & =\text { neoadjuvant chemotherapy } \\ \mathrm{UTI} & =\text { urinary tract infection }\end{aligned}$

